AIAA-98-2881

\title{
IMPORTANT SCALING PARAMETERS FOR TESTING MODEL-SCALE HELICOPTER ROTORS
}

\author{
Jeffrey D. Singleton* and William T. Yeager, Jr. ${ }^{\dagger}$ \\ U.S. Army Research Laboratories, Vehicle Technology Center \\ NASA Langley Research Center \\ Hampton, VA
}

\begin{abstract}
An investigation into the effects of aerodynamic and aeroelastic scaling parameters on model scale helicopter rotors has been conducted in the NASA Langley Transonic Dynamics Tunnel. The effect of varying Reynolds number, blade Lock number, and structural elasticity on rotor performance has been studied and the performance results are discussed herein for two different rotor blade sets at two rotor advance ratios. One set of rotor blades were rigid and the other set of blades were dynamically scaled to be representative of a main rotor design for a utility class helicopter. The investigation was conducted in forward flight at rotor advance ratios of 0.15 and 0.35. Additionally, the rotors were tested over a range of nominal test medium densities from 0.00382 slugs $/ \mathrm{ft}^{3}$ to 0.009 slugs $/ \mathrm{ft}^{3}$. This range of densities permits the acquisition of data for several Reynolds and Lock number combinations.
\end{abstract}

\section{Nomenclature}

Positive directions for forces, moments, angles, and velocities are shown in Figure 1.

\footnotetext{
A balance axial force, $l b s$

a speed of sound, $\mathrm{ft} / \mathrm{sec}$

$a_{0} \quad$ airfoil section lift curve slope

*Research Engineer, Aeroelasticity Branch

${ }^{\dagger}$ Senior Research Engineer, Aeroelasticity Branch

This paper is declared a work of the U.S. Government and is not subject to copyright protection in the United States.
}

\begin{tabular}{|c|c|}
\hline$C_{D}$ & rotor drag coefficient, $D / \rho \pi R^{2}(\Omega R)^{2}$ \\
\hline$C_{L}$ & rotor lift coefficient, $L / \rho \pi R^{2}(\Omega R)^{2}$ \\
\hline$C_{Q}$ & rotor torque coefficient, $Q_{R} / \rho \pi R^{3}(\Omega R)^{2}$ \\
\hline$c$ & nominal blade chord, $f t$ \\
\hline$d$ & rotor diameter, $f t$ \\
\hline$D$ & rotor drag, $l b s, D=N \sin \alpha_{s}+A \cos \alpha_{s}$ \\
\hline$e$ & flapping hinge offset, percent radius \\
\hline$I_{b}$ & blade mass moment of inertia about \\
\hline & flapping hinge, slug $-f t^{2}, \int_{e}^{R} m r^{2} d r$ \\
\hline$L$ & rotor lift, $l b s, L=N \cos \alpha_{s}-A \sin \alpha_{s}$ \\
\hline$M_{t i p}$ & rotor tip Mach number in hover, $\Omega R / a$ \\
\hline$M_{1,90}$ & rotor tip Mach number at $\psi=90^{\circ}$ \\
\hline$N$ & balance normal force, $l b s$ \\
\hline$Q_{R}$ & rotor shaft torque, $f t-l b$ \\
\hline$R$ & rotor radius, $f t$ \\
\hline$r$ & $\begin{array}{l}\text { spanwise distance along blade radius } \\
\text { from center of rotation, } f t\end{array}$ \\
\hline Re & Reynolds number, per foot, $\rho V / \mu_{o}$ \\
\hline$R e_{1,90}$ & $\begin{array}{l}\text { rotor tip Reynolds number at } \\
\psi=90^{\circ} \text {, per foot }\end{array}$ \\
\hline$V$ & free-stream velocity, $\mathrm{ft} / \mathrm{sec}$ \\
\hline$z$ & $\begin{array}{l}\text { distance from wind-tunnel floor to rotor } \\
\text { plane of rotation, } f t\end{array}$ \\
\hline$\alpha_{s}$ & rotor shaft angle of attack, degrees \\
\hline$\gamma$ & rotor blade Lock number, $\rho a_{o} c R^{4} / I_{b}$ \\
\hline$\theta$ & $\begin{array}{l}\text { rotor blade collective pitch angle at } \\
r / R=0.75 \text {, degrees }\end{array}$ \\
\hline$\theta_{1}$ & $\begin{array}{l}\text { twist angle built into rotor blade, } \\
\text { positive nose up, degrees }\end{array}$ \\
\hline$\mu$ & rotor advance ratio, $V / \Omega R$ \\
\hline$\mu_{0}$ & viscosity, $l b-\sec / f t^{2}$ \\
\hline$\rho$ & test-medium mass density, slugs $/ \mathrm{ft}^{3}$ \\
\hline$\psi$ & rotor blade azimuth angle, degrees \\
\hline$\Omega$ & rotor rotational velocity, radians/sec \\
\hline
\end{tabular}




\section{Introduction}

In general, the development of any new aircraft and in particular a new helicopterF rotor system requires large amounts of analysis and testing. As rotor technology has developed, new rotor systems have become increasingly complex. Today's newer rotor systems often include hingeless or bearingless hubs, the rotor blades incorporate unique planform and twist geometries, as well as utilize advanced airfoils. Therefore, it is desirable to test model-scale rotors to verify a candidate design before committing large amounts of resources to full-scale design verification testing. The use of model-scale rotors to achieve this design verification is cost effective and also permits a much easier variation of model parameters to conduct design studies and optimizations. However, in order to obtain the maximum benefit from model-scale testing of helicopter rotor systems, one must pay great attention to the aerodynamic environment in which the model is to be tested. When testing a model-scale rotor system, some compromises will have to be made. It is up to the model designer and test engineer to determine which parameters are most important. In considering the calculation of rotor performance coefficients for a model-scale rotor, the most important parameters are those involved with matching the correct aerodynamic forces, namely Mach number and Reynolds number. Rotor blade Lock number ensures that the rotor has the correct aerodynamic damping and aerodynamic coupling characteristics. ${ }^{1}$ Thus, for the measurement of rotor loads, Lock number and rotor blade elasticity should also be considered.

For performance testing at model scale, matching the full-scale tip Mach number is required to duplicate compressibility effects and also to minimize the reduction in Reynolds number. The importance of simulating the correct tip Mach number is especially dependent upon the rotor airfoils selected due to the relatively high Mach numbers encountered by the advancing blade. In order to match full-scale tip Mach number, one must operate the model rotor at the same tip speed as the full-scale vehicle if tested in air. The reduced scale of the model leads to much higher rotor speed to achieve the desired tip Mach number. This means that the rotational velocity of the scale-model must be multiplied by the reciprocal of the geometric scale factor. For example, the rotational velocity of a $1 / 5$ th size model-scale rotor would be 5 times that of the full-scale helicopter.
This in turn leads to high centrifugal loads on the model. Additionally, it is not possible to match both full-scale tip Mach numbers and Reynolds numbers with a model-scale rotor being tested in air at atmospheric pressures and density. Thus, it can be seen that attempting to develop and test a model-scale helicopter rotor is not such an easy task. But what are some of the effects of matching or not matching full-scale values of the key parameters?

The importance of Reynolds number in considering flow similarity has been well established in fixed wing aerodynamics. However, it's effect on rotary wing aerodynamics is not as well understood. As of 1972, few comparisons had been done between full-scale data and model-scale data. ${ }^{2}$ As recently as 1985 , Carr $^{3}$ states that little had been done to determine the influence of Reynolds number on dynamic stall, since it is difficult to vary Reynolds number significantly without introducing compressibility effects as well. Unsteadiness can also have a significant effect on transition. Therefore, proper representation of the Reynolds number effect on dynamic stall remains an important, and presently unsolved, question. This further raises a question about the accuracy of rotor performance estimates at the extreme edges of a rotor's operating envelope

A few effects of Reynolds number on model-scale rotor testing are known. Keys, ${ }^{4}$ states that in air, even though model rotors are tested at full-scale tip Mach numbers, the Reynolds number is low by the ratio of the geometric scale factor. This is the primary cause of differences between full-scale rotor performance and model-scale rotor test data. The difference between model-scale and full-scale performance data consists of an incremental profile power variation at zero thrust and additional profile power increment which is a function of the lift coefficient. Another example of the variation in profile power with lift coefficient and Reynolds number occurs in models with tapered tips. The very low Reynolds number of the tapered tip can cause premature separation that does not occur at full-scale. In forward flight, unsteady aerodynamic stall delay effects are much larger at model-scale than at full-scale. So, it has been shown that even though model-scale rotors can be tested at full-scale tip Mach numbers, the lower Reynolds number at model-scale can have a powerful effect on the measurement of rotor performance coefficients. 
According to Bingham and Kelley, ${ }^{5}$ the effects of Reynolds number on the performance of scaled model rotors increases with increasing forward flight velocity and decreases with increasing tip chord of non-rectangular blades. The Reynolds number influences become most significant as the retreating blade airfoil sections approach or exceed the maximum lift coefficients characteristic of model-scale Reynolds number. Induced power benefits should not be significantly altered by Reynolds number variations, but the influence on profile power is substantial. However, Reynolds number influences at higher advance ratios or thrust coefficients for Bingham and Kelley's investigation did not permit direct experimental verification of the above conclusion. This lack of verification resulted in concern that design opportunities may be overlooked if model-scale test results are not properly evaluated.

One method of achieving full scale tip Mach numbers while also obtaining relatively high Reynolds number for a scale model is by using a heavy gas test medium. ${ }^{6}$ Yeager and Mantay showed that Mach number effects on model rotor data obtained in Refrigerant-12 (R-12) are essentially the same as full-scale rotor aerodynamic performance data obtained in air. ${ }^{7}$ Yeager and Mantay also indicated that the Reynolds number effects might be minor in rotor aerodynamic performance testing compared to the combined effects of rotor solidity and blade elastic properties. Therefore, blade elastic modeling should also be considered a significant parameter in model-scale rotor aerodynamic performance testing. Finally, to fully model the complex aerodynamic environment of a helicopter rotor system, some attention must be paid to the rotor blade Lock number, $\gamma$, which is defined as the ratio of the blade aerodynamic forces to the blade inertia forces. Correct scaling of rotor Lock number is important for the prediction of rotor loads and stability. However, its contribution to the rotor aerodynamic environment can not be neglected as it directly affects blade flapping angles. Still, it would be desirable to isolate the various effects of Reynolds number, Lock number, and blade elasticity so as to more fully understand their total effect upon predicting full scale helicopter rotor performance and dynamic loads from scale-model rotor tests.

Even at the moderately high Mach numbers in which a typical helicopter rotor operates, the effect of Reynolds number can be significant. These effects upon maximum lift coefficient are illustrated in Figure 2 for a 63 series airfoil section. ${ }^{11}$ The Reynolds number effect is also apparent in plots of minimum section drag coefficients. The range of Reynolds numbers achieved by testing in a heavy gas is indicated on Figure 2 as well as the typical range for model-scale rotor blades tested in air at atmospheric pressure.

A study has been conducted in the NASA Langley Transonic Dynamics Tunnel which investigated the isolated and combined effects of varying several aerodynamic and dynamic scaling parameters. ${ }^{8}$ These parameters were Reynolds number, rotor blade Lock number, and blade elasticity. Two sets of geometrically similar rotor blades were tested: a rigid blade set and a set of blades which were dynamically scaled to be representative of the main rotor of a utility class helicopter (e.g. the U.S. Army UH-60 Blackhawk helicopter). This paper presents some forward flight results of that study pertinent to how the variation in Reynolds number, Lock number, and blade structural elasticity affects the performance measurements for a model-scale helicopter rotor system.

Test Apparatus and Procedures

The data presented herein were obtained via the Aeroelastic Rotor Experimental System (ARES) and the NASA Langley Transonic Dynamics Tunnel (TDT). The ARES is a fly-by-wire belt-driven rotor system testbed which is used to experimentally test dynamically scaled models of up to approximately nine feet in diameter. The Transonic Dynamics Tunnel incorporates the use of a heavy gas test medium to permit the testing of scale model aircraft at relatively high densities (thus higher Reynolds number), lower speed of sound (and thus higher Mach numbers), and subtle changes in the ratio of specific heats as well as viscosity. These differences ease the manufacturing requirements for building a set of model-scale rotor blades. ${ }^{9}$

\section{$\underline{\text { Wind Tunnel }}$}

The TDT is a continuous-flow tunnel with a slotted test section capable of operation up to Mach 1.2 at stagnation pressures of 0.1 to 1.0 atmosphere. The tunnel test section is 16 feet square with cropped corners and has a cross-sectional area of $248 \mathrm{ft}^{2}$. Cur- 
rently, either air or refrigerant-134a (R-134a) may be used as the test medium. At the time that these data were taken, the Transonic Dynamics Tunnel used refrigerant-12 (R-12) as the test medium. For this study, data were taken over a range of tunnel operating densities from 0.00382 slugs $/ \mathrm{ft}^{3}$ to 0.009 slugs $/ \mathrm{ft}^{3}$. Because of its high density at normal atmospheric pressure and low speed of sound, the use of R-12 aids the matching of model-scale rotor Mach number to full-scale values and provides Reynolds numbers greater than that obtainable using air. Furthermore, some restrictions on model structural design parameters are eased while maintaining dynamic similarity. The heavier test medium permits a simplified structural design to obtain the required stiffness characteristics, and thus eases design and fabrication requirements of the model. ${ }^{9}$

Aeroelastic Rotor Experimental System

The ARES has a streamlined fuselage enclosing the rotor controls and drive system. The ARES is powered by a variable frequency synchronous motor rated at 47 horsepower output at 12,000 RPM. The motor is connected to the rotor shaft through a belt-driven two-stage speed reduction system. The ARES rotor control system and rotor shaft angle of attack are remotely controlled from the wind tunnel control room. The model rotor shaft angle of attack is varied by an electrically controlled hydraulic actuator. Blade collective pitch and lateral and longitudinal cyclic pitch are input to the rotor shaft through a swashplate. The swashplate is moved by three hydraulic actuators.

Instrumentation on the ARES allows continuous displays of model rotor control settings, rotor moments and forces, blade structural moments, and pitch link loads. The ARES rotor shaft pitch attitude is measured by a static accelerometer, and rotor control positions are measured by linear potentiometers connected to the swashplate. Rotor blade flapping and lagging are measured by rotary potentiometers mounted on the rotor hub and geared to the rotor cuff. Rotor shaft speed is determined by a magnetic sensor. The rotating blade data are transferred through a 30-channel slip-ring assembly. Rotor forces and moments are measured by a sixcomponent strain-gage balance mounted below the rotor pylon and drive system. The balance is fixed with respect to the rotor shaft and pitches with the fuselage and by design, fuselage forces and moments are not sensed.

\section{Rotor Blades and Hub}

The model rotor hub used in this investigation is a four-bladed articulated hub with coincident lead-lag and flapping hinges. The hub was operated with a pitch-flap coupling ratio of 0.5 (flap up, pitch down). The attachment point of the blade pitch link was 1.4 inches aft of the blade pitch axis.

Two blade sets were used for this evaluation and both blade sets were $1 / 6$-size and Mach-scaled representations of UH-60A rotor blades. The first blade set was a dynamically scaled (elastic) version of the UH-60A rotor. The second blade set was designed to be approximately four times more stiff in flapwise bending and approximately twice as stiff in chordwise bending and torsion as the elastic blade set. These blades are referred to as the "rigid" blade set. The dynamic characteristics of the rigid blade set do not represent actual helicopter blades in terms of flapwise (out-of-plane), chordwise (in-plane), or torsional stiffness. They were included in the investigation solely to isolate the effects of structural elasticity. Both blade sets were untapered with a $20^{\circ}$ swept tip with sweep initiating at the 94-percent-radius station and used SC1095 and SC1095-R8 airfoils (Figure 3). Aerodynamic characteristics of these airfoils are documented by Noonan. ${ }^{10}$ The area, thrust-weighted, and torque-weighted solidities for the rotor were each 0.0825 . Planform geometry and twist distribution of these blades are shown in Figure 3 . One blade of each blade set was instrumented with resistance-wire strain-gage bridges calibrated to measure blade structural moments. These gages were used to monitor limit loads for safety considerations. Embedded in each rigid blade were four hollow steel tubes, two extending along the leading edge and two along the trailing edge of the blade spar centered about the quarter-chord. These tubes allowed for distributed non-structural mass to be added to the blades from the blade root to 80 percent radius. Steel or tungsten rods were inserted into these tubes to ballast the blade to obtain the desired Lock number for the tunnel test medium operating density. 
Testing Methods and Data Reduction

The focus of this investigation was to examine the effects of Mach number, Lock number, Reynolds number, and dynamic scaling upon rotor performance. Therefore, both blade sets were evaluated over the same range of nominal test conditions defined by tip Mach number, $M_{\text {tip }}$, rotor lift coefficient, $C_{L}$ and rotor drag coefficient or propulsive force, $C_{D}$. Each blade set was ballasted for a specific test medium density. At each test point, the rotor rotational speed and tunnel conditions were adjusted to give the desired values of $M_{\text {tip }}$ and rotor advance ratio, $\mu$. Blade collective pitch, $\theta$, and shaft angleof-attack, $\alpha_{s}$ were then swept to obtain variations in rotor lift and propulsive force. At each collective pitch and shaft angle setting, the cyclic pitch was used to remove rotor first-harmonic flapping with respect to the rotor shaft and then data were recorded. The maximum value of collective pitch attained at each shaft angle of attack was determined in most cases by either blade load limits or the ARES drive system limits. Rotor aerodynamic performance and blade loads were measured in forward flight at two advance ratios for $\alpha_{s}$ from $0^{\circ}$ to $-11.8^{\circ}$. Variations in Reynolds number and Lock number were achieved by varying the tunnel operating density and/or blade ballast.

Model dead weight tares were determined throughout the range of shaft angles of attack with the blades on and with them removed for each configuration of blade ballast. Aerodynamic rotor hub tares were determined with the blades removed throughout the ranges of shaft angle of attack and advance ratios investigated. Both dead weight and aerodynamic hub tares have been removed from the data presented herein. All data were acquired at $z / d$ equal to 0.87 . No correction has been applied to the data to account for tunnel wall effects; however, for the flight conditions tested these effects have been shown to be small. ${ }^{6}$ All strain-gage and balance voltage readings were zeroed with the blades resting on the down stops and non-rotating prior to each test run. At each test point, tunnel parameter data were averaged and stored digitally. Performance data, i.e. fixed system forces and moments, were averaged and stored as digital counts. At the completion of each run, all strain-gage and balance voltage readings were again recorded with the blades resting on the down stops and non-rotating. These final voltage readings were used to correct for any amplifier voltage drift.

The quality of the performance data obtained during this investigation with regards to repeatability was addressed. During the test, 52 target data points were randomly selected to be repeated. The total number of actual repeat points was 102 . The average deviation in $C_{L}, C_{D}$, and $C_{Q}$ was determined from the differences between selected target values and the repeated values. The average deviations for constant values of $\mu, \alpha_{s}, \theta$, and rotor cyclic pitch were determined to be as follows:

$$
\begin{aligned}
& C_{L} \pm 0.00004 \\
& C_{D} \pm 0.00001 \\
& C_{Q} \pm 0.00001
\end{aligned}
$$

$\underline{\text { Discussion of Results }}$

Based on the results reported by Yeager and Mantay, the first parameter to be studied was the effect of rotor blade elasticity upon rotor performance coefficients. As seen in Figure 4, a small effect of varying blade stiffness is seen at low values of rotor $C_{L}$. However, at higher advance ratios this effect is not apparent (Figure 5). As noted, the results dealing with "rigid versus elastic" blade sets presented herein are different than those which were originally presented by Yeager and Mantay. ${ }^{7}$ This may be caused by the relative differences between "rigid blades" in each case. The original study states that the "rigid" blades were an order of magnitude more stiff than the baseline blades, while in the case of this investigation, the "rigid" blades were only 2-4 times more stiff. There may also be differences due to the fact that the earlier configuration was a teetering rotor while this configuration is an articulated configuration.

Because of the small effects due to blade elasticity determined in this investigation it was decided to examine the effects of variations in Reynolds number and Lock number through data obtained via the rigid blade set. The reason being is that this particular set of data were taken over a greater range of test medium density and hence a wider range Reynolds number and rotor blade Lock number. Over the range of test medium densities utilized, the Reynolds number of the advancing blade tip increases from approximately 5.3 to 14.1 million per foot and Lock number increases from 9.4 to 15.1 . 
As test medium density is increased, the Reynolds number seen by the blade increases. If the blades are not re-ballasted to give the desired Lock number then we see results such as those shown in Figure 6 and Figure 7 taken at $\mu=0.15$. As can be seen in these figures, there is no apparent effect of increasing Reynolds number, but Lock number is also increasing. The data presented for $\mu=0.15$ were taken at a constant $\alpha_{s}=-1.8^{\circ}$. By isolating the effects of varying Lock number while maintaining a constant Reynolds number, it can be seen that at a higher Lock number there is a definite and significant increase in rotor $C_{Q}$ and $C_{D}$ at a given $C_{L}$ (Figure 8 and Figure 9). Conversely, if a constant Lock number is maintained and Reynolds number is increased, then the expected decrease in rotor $C_{Q}$ at a given $C_{L}$ is noted (Figure 10 and Figure 11). These trends were repeated at $\mu=0.35$ as shown in Figure 12 through Figure 17. The data presented for $\mu=0.35$ were taken at a constant $\alpha_{s}=-5.0^{\circ}$.

The question may be raised as to whether or not experimental results dealing with differences in Lock number agree with theory? The answer appears to be "yes". Based on a cursory examination of trends based on equations developed from blade element theory and presented in Gessow and Myers, ${ }^{12}$ the trends in $C_{Q}$ at constant $C_{L}$ are in agreement. To be more specific, the equations show that an increase in Lock number will result in an increase in $C_{Q}$ and the data show the same trends. Reynolds number trends in the data also agree with theory. Rotor torque decreases with increased Reynolds number indicating a decrease in rotor blade profile drag (Figure 10 and Figure 16). The effect of Reynolds number is also seen in the decrease in rotor propulsive force with increased advance ratio indicating a reduction in blade drag on the retreating side of the rotor disk where viscous effects should dominate (Figure 11 and Figure 17).

Another way of looking at the data is to examine the effect of varying Lock and Reynolds number for a specific rotor task as defined by constant $C_{L}$ (Figure 18 through Figure 23). These data are obtained by cross plotting from rotor $C_{L}$ versus $C_{Q}$ and $C_{L}$ versus $C_{D}$ curves to generate rotor $C_{D}$ versus rotor $C_{Q}$. Travel along the curves represent an increase in $\mu$ from 0.15 to 0.35 as propulsive force $C_{D}$ increases (becomes more negative). Figure 18 through Figure 20 illustrate data taken at constant tunnel operating density while Figure 21 through Figure 23 show data taken at constant Lock number. Once again it is evident that both Lock and Reynolds number effects are significant at model-scale. At a specified rotor task, increasing Lock number increases rotor torque required. Figure 21 through Figure 23 are more complex as Reynolds number is increased due to both $\mu$ and increasing tunnel operating density. It is important to note that at higher advance ratios the torque decrease due to increasing tunnel operating density is greater than at lower advance ratios as expected.

\section{$\underline{\text { Conclusions }}$}

In conclusion, it has been shown that Reynolds number and Lock number effects are very important to the testing of model-scale rotor systems for helicopter rotor performance. It is known that it is not possible to simultaneously match all key full-scale aerodynamic parameters. The best course of action for testing model-scale rotors is to match the tip Mach number and test at as high a Reynolds number as is feasible and at the full-scale value of Lock number. Testing model-scale rotors in a heavy gas environment has also proven very successful, particularly testing rotors in R-12. It is anticipated that the merit of testing in heavy gas will continue with the next generation of refrigerant, R-134a. The data presented herein support the following conclusions:

1. Reynolds number effects are important when testing model scale rotor systems. A decrease in rotor $C_{Q}$ is indicated at a given value of rotor $C_{L}$ when testing at higher Reynolds numbers.

2. Lock number is also an important parameter when measuring rotor performance coefficients. An increase in rotor $C_{Q}$ is indicated at a given value of rotor $C_{L}$ when testing at higher Lock numbers.

3. It may be possible to offset performance losses caused by low Reynolds number testing by adjusting Lock number for model-scale rotors, but further testing would be necessary to investigate this phenomena more fully.

In summary, through careful attention to model scaling parameters and test conditions, it is desirable to test new helicopter rotor systems at model-scale to 
prove or disprove new design concepts before moving on to full-scale development where the cost of parametric variation of a design is often prohibitively expensive.

\section{$\underline{\text { References }}$}

${ }^{1}$ Bielawa, Richard L.:Rotary Wing Structural Dynamics and Aeroelasticity, AIAA Education Series, Washington, D.C., 1992.

${ }^{2}$ Hardy, W. G. S.: "The Effects of Reynolds Number on Rotor Stall", U.S. Army TN A74-22451, 1972.

${ }^{3}$ Carr, L. W.: "Dynamic Stall Progress in Analysis and Prediction", U.S. Army TN A85-47026, 1985.

${ }^{4}$ Keys, C. N.; McVeigh, Michael A.; Dadone, Leo; McHugh, Francis J.: "Considerations in the Estimation of Full-Scale Rotor Performance from Model Rotor Test Data", Presented at the 39th Annual Forum of the AHS, St. Louis, MO, May 1983.

${ }^{5}$ Bingham, G. J.; Kelley, Henry L.: "Reynolds Number Influences on the Aerodynamic Performance of Model-scale Rotor Blades", Presented at the AHS National Specialists Meeting on Helicopter Test Technology, Williamsburg, VA, November 1984 .

${ }^{6}$ Mantay, Wayne R.; Yeager, William T., Jr.; Hamouda, M-Nabil, Cramer, Robert G., Jr.; and Langston, Chester W.: "Aeroelastic Model Helicopter Rotor Testing in the Langley TDT", NASA TM-86440, U.S. Army AVSCOM TM 85-B-5, 1985.

${ }^{7}$ Yeager, William T., Jr.; Mantay, Wayne R.: “Correlation of Full-Scale Helicopter Rotor Performance in Air with Model-scale Freon Data", NASA TN D8323,1976 .

${ }^{8}$ Singleton, Jeffrey D.; Yeager, William T., Jr.; and Wilbur, Matthew L.: "Performance Data From a Wind-Tunnel Test of Two Main-Rotor Blade Designs for a Utility-Class Helicopter", NASA TM 4183, U.S. Army AVSCOM TM 90-B-004, June 1990.

${ }^{9}$ Lee, Charles: "Weight Considerations in Dynamically Similar Model Rotor Design”, SAWE Paper No. 659, May 1968.

${ }^{10}$ Noonan, Kevin W. and Bingham, Gene J.: “Aerodynamic Characteristics of Three Helicopter Rotor Airfoil Sections at Reynolds Numbers from Model
Scale to Full Scale at Mach Numbers from 0.35 to 0.90", NASA TP-1701, U.S. Army AVRADCOM TR 80-B-5, 1980.

${ }^{11}$ Prouty, R. W.:Helicopter Aerodynamics, PJS Publications, Peoria, IL, 1985.

${ }^{12}$ Gessow, Alfred and Myers, Garry C.;Aerodynamics of the Helicopter, Frederick Ungar Publishing CO., New York, 1952.

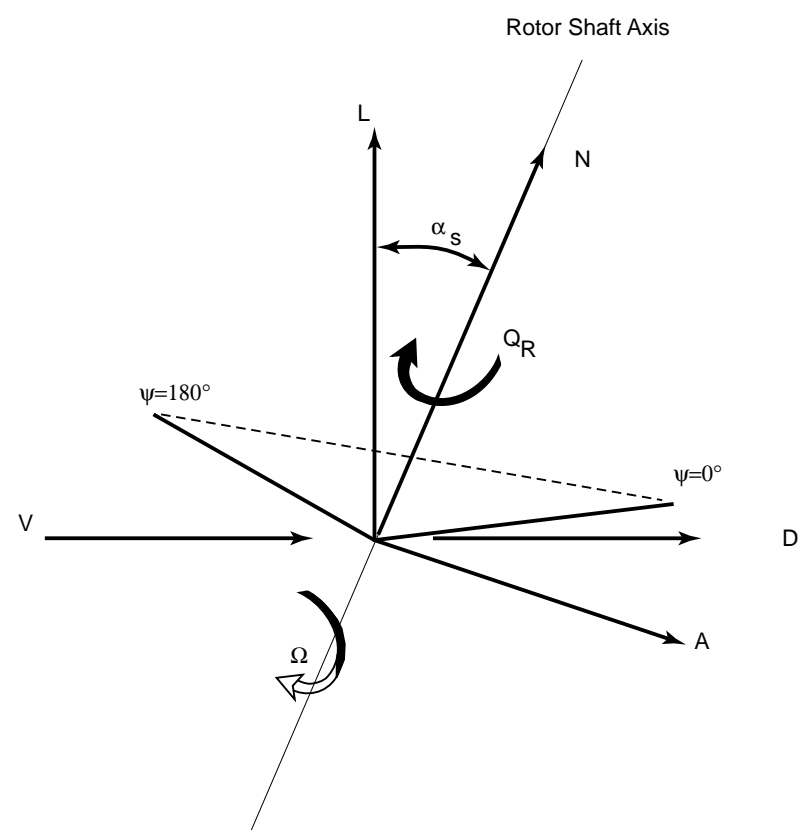

Figure 1: Notation showing positive directions for forces, moments, angles, and velocities.

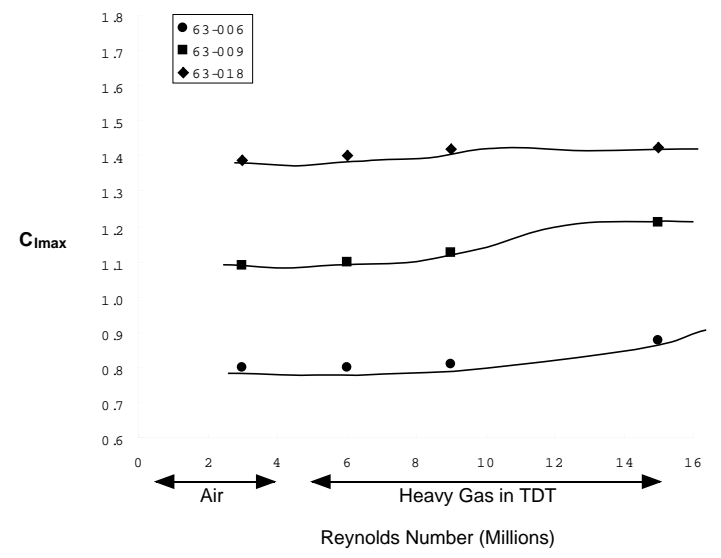

Figure 2: Maximum lift coefficient vs. Reynolds number (per foot). 

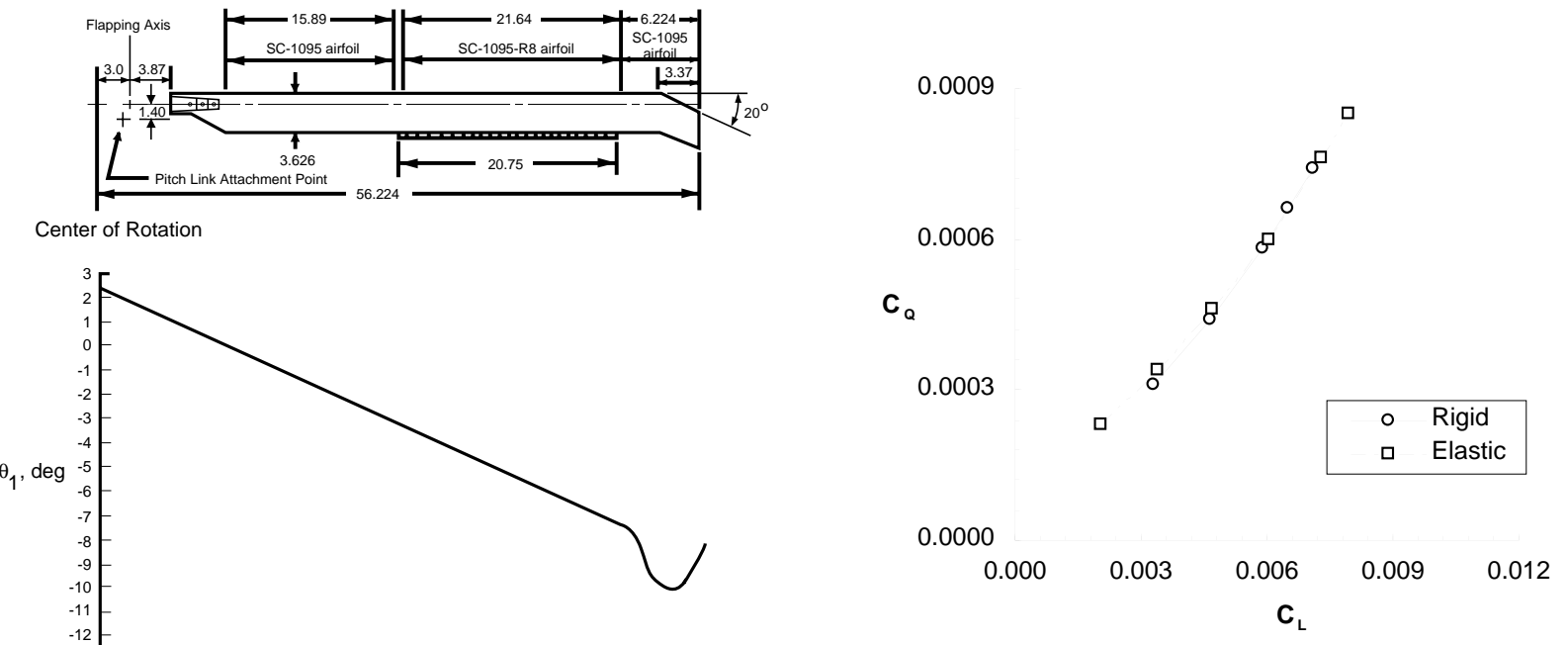

Figure 5: Effect of rotor blade elasticity at $\mu=0.35$ and $\alpha_{s}=-1.8^{\circ}$.

Figure 3: Model Helicopter Rotor Blades.

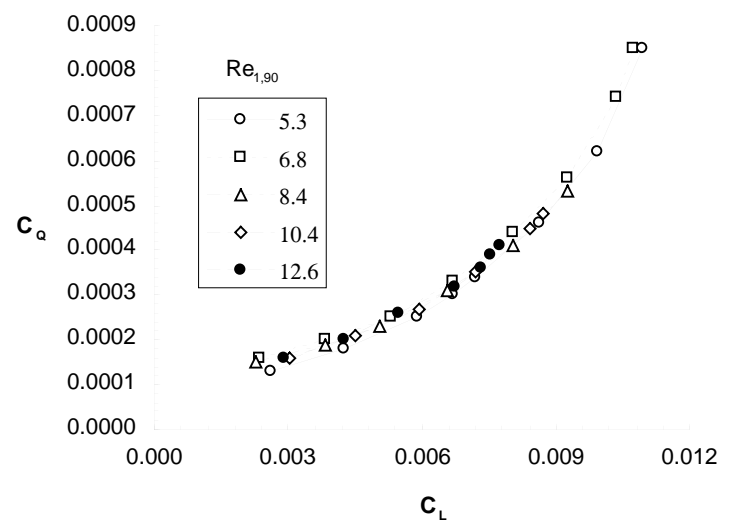

Figure 6: Reynolds number effect on rotor torque at $\mu=0.15$ with varying Lock number.

Figure 4: Effect of rotor blade elasticity at $\mu=0.15$ and $\alpha_{s}=-1.8^{\circ}$. 


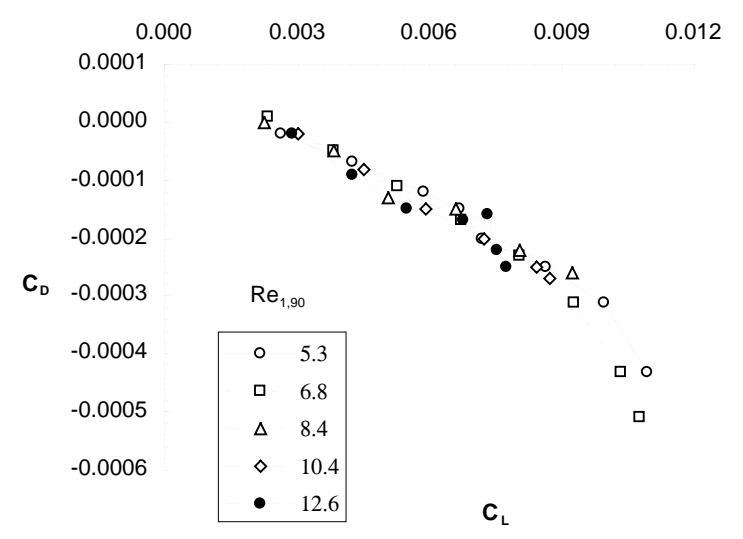

Figure 7: Reynolds number effect on rotor drag at $\mu=0.15$ with varying Lock number.

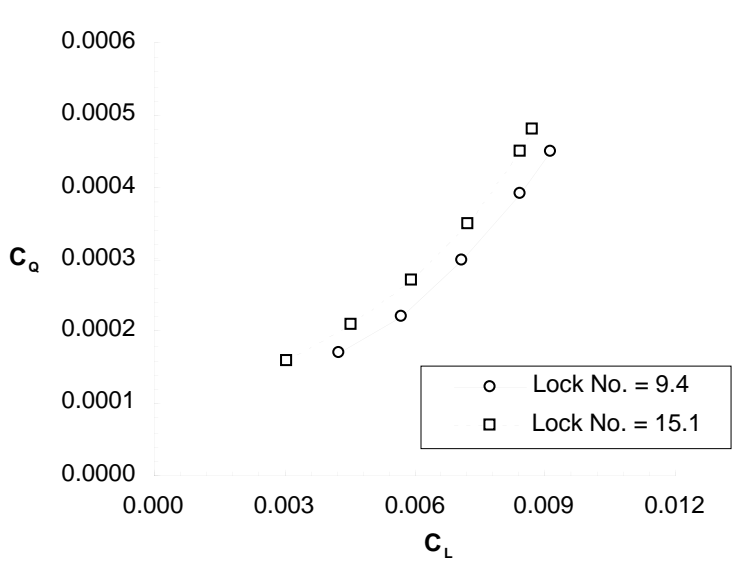

Figure 8: Lock number effect on rotor torque at $\mu=$ 0.15 at constant Reynolds number.

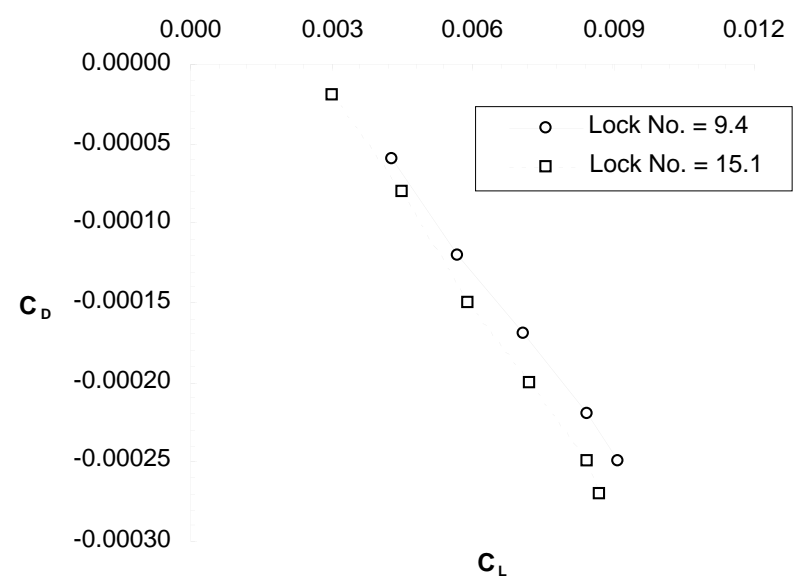

Figure 9: Lock number effect on rotor drag at $\mu=$ 0.15 at constant Reynolds number.

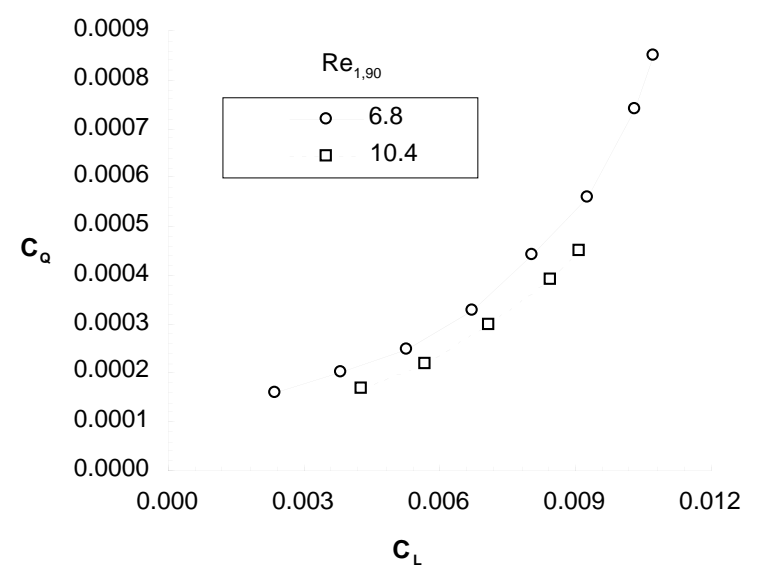

Figure 10: Reynolds number effect on rotor torque at $\mu=0.15$ at constant Lock number.

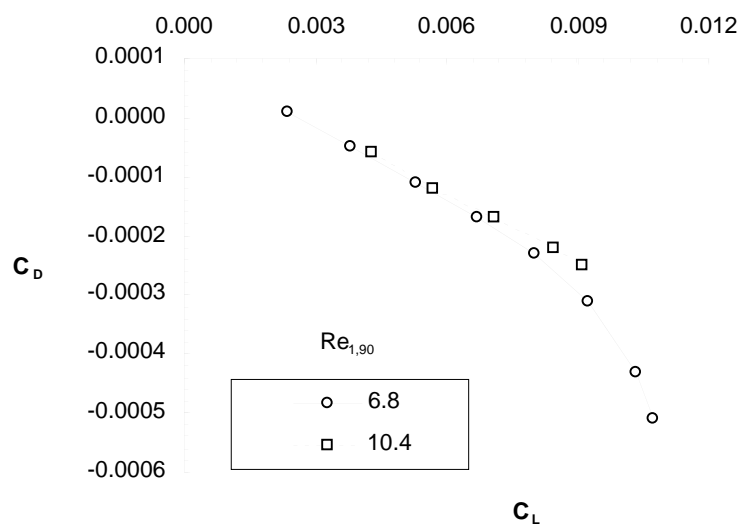

Figure 11: Reynolds number effect on rotor drag at $\mu=0.15$ at constant Lock number.

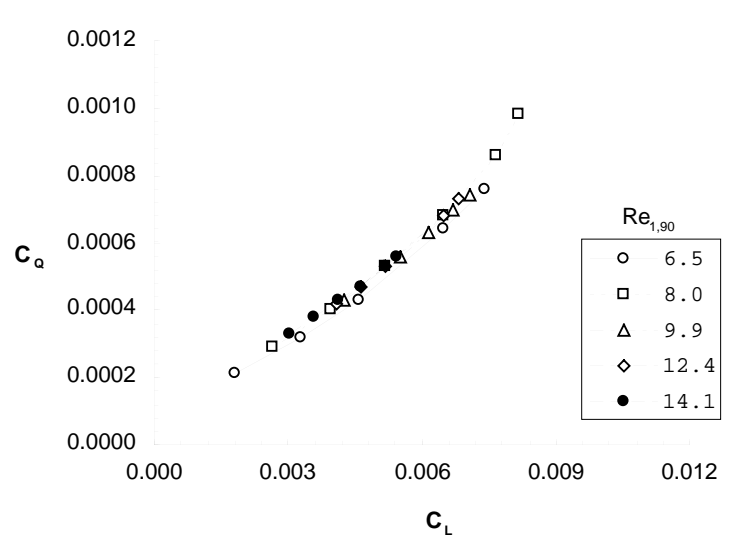

Figure 12: Reynolds number effect on rotor torque at $\mu=0.35$ with varying Lock number. 


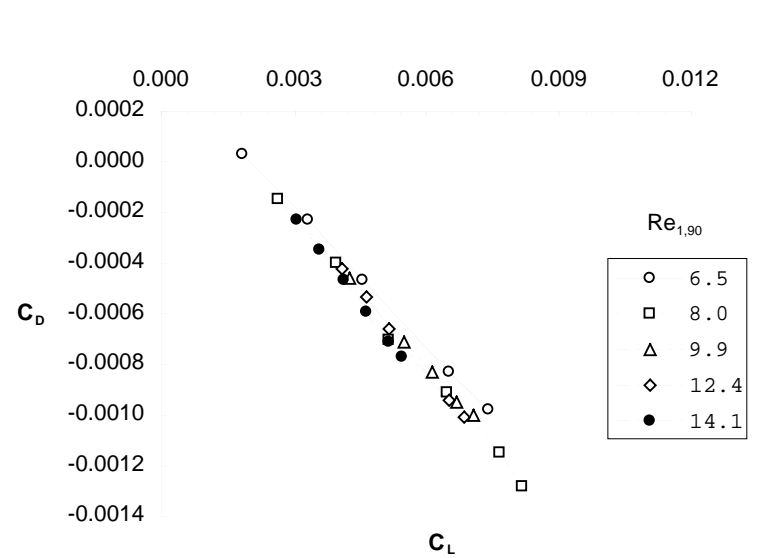

Figure 13: Reynolds number effect on rotor drag at $\mu=0.35$ with varying Lock number.

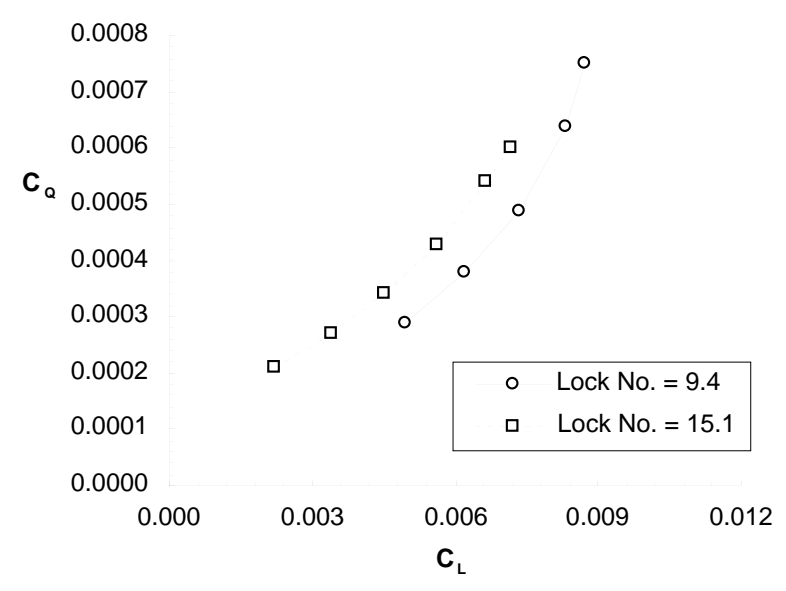

Figure 14: Lock number effect on rotor torque at $\mu=0.35$ at constant Reynolds number.

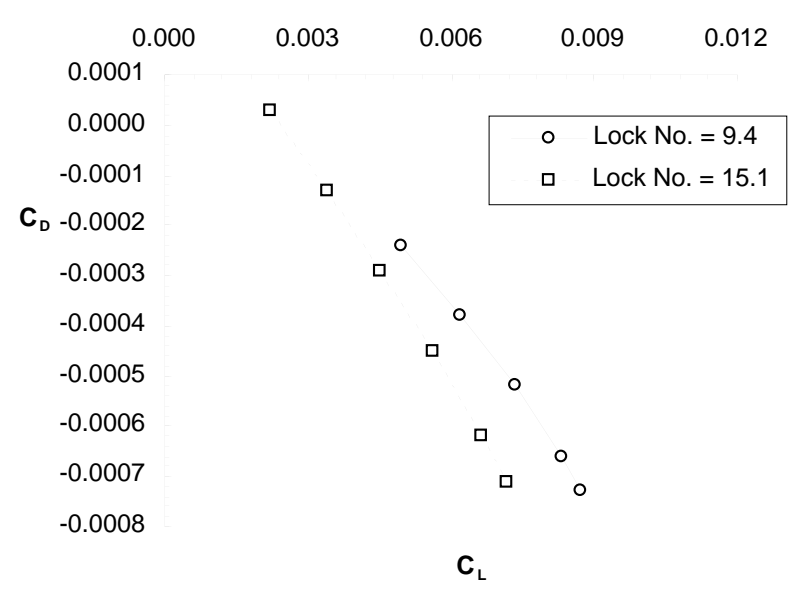

Figure 15: Lock number effect on rotor drag at $\mu=$ 0.35 at constant Reynolds number.

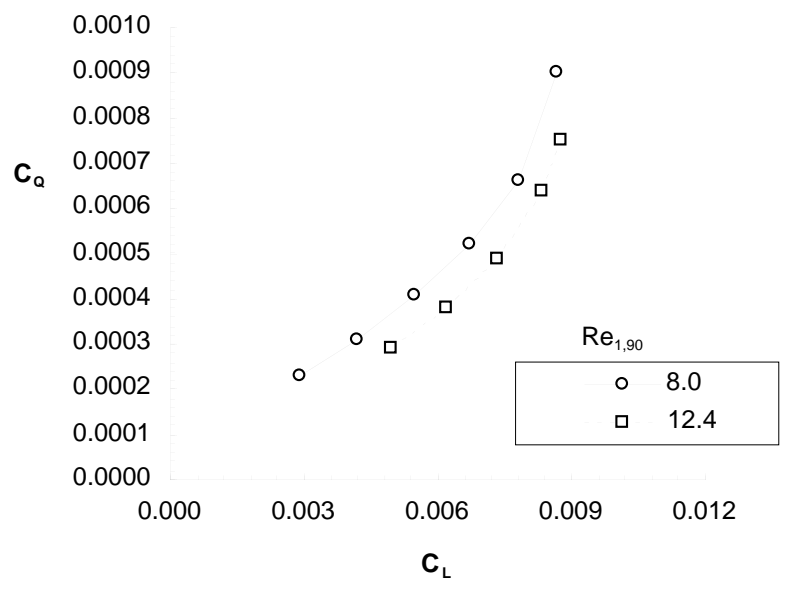

Figure 16: Reynolds number effect on rotor torque at $\mu=0.35$ at constant Lock number.

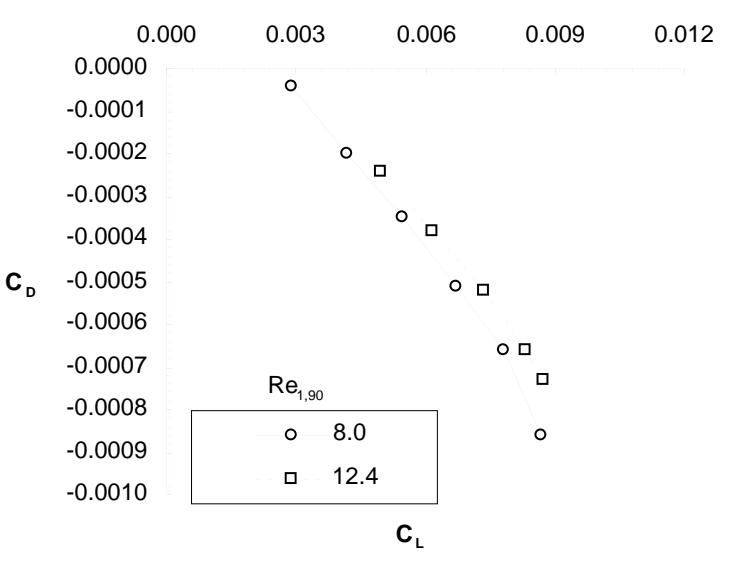

Figure 17: Reynolds number effect on rotor drag at $\mu=0.35$ at constant Lock number.

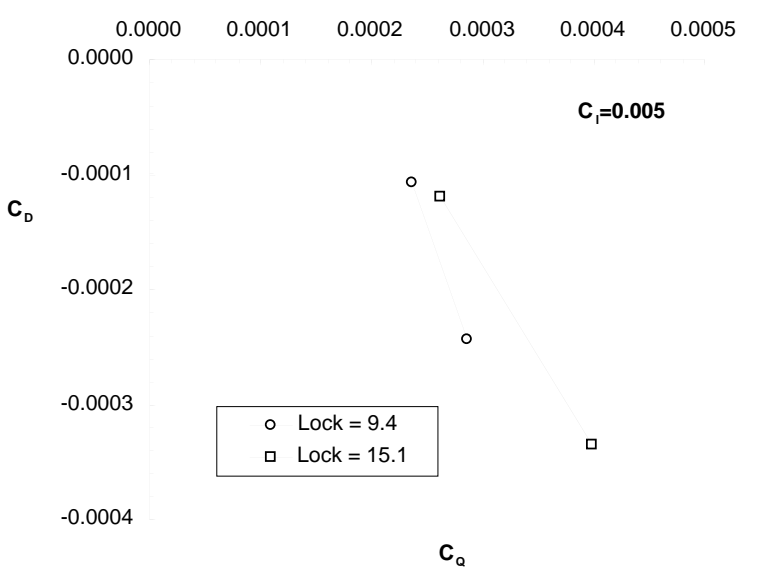

Figure 18: Effect of Lock number at $C_{L}=0.005$. 


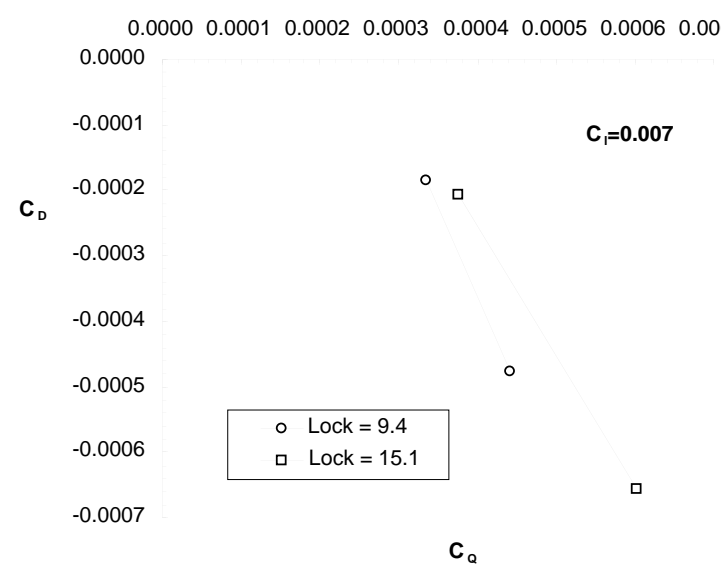

Figure 19: Effect of Lock number at $C_{L}=0.007$.

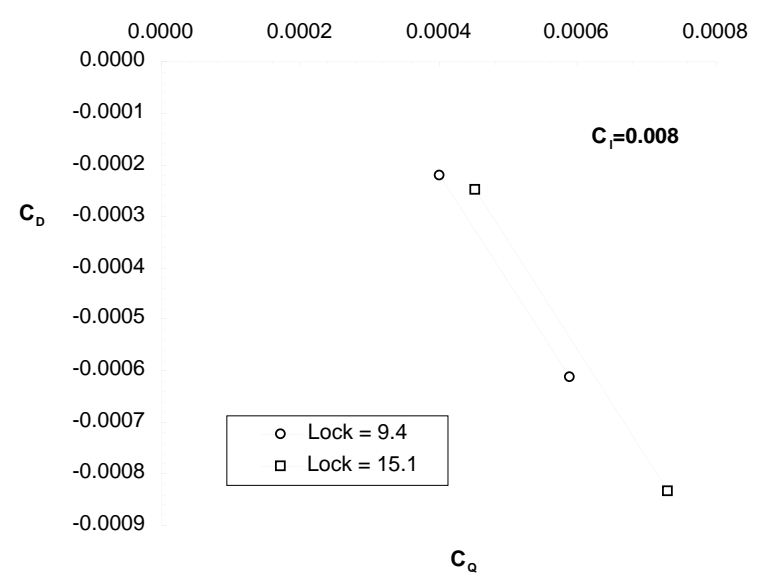

Figure 20: Effect of Lock number at $C_{L}=0.008$.

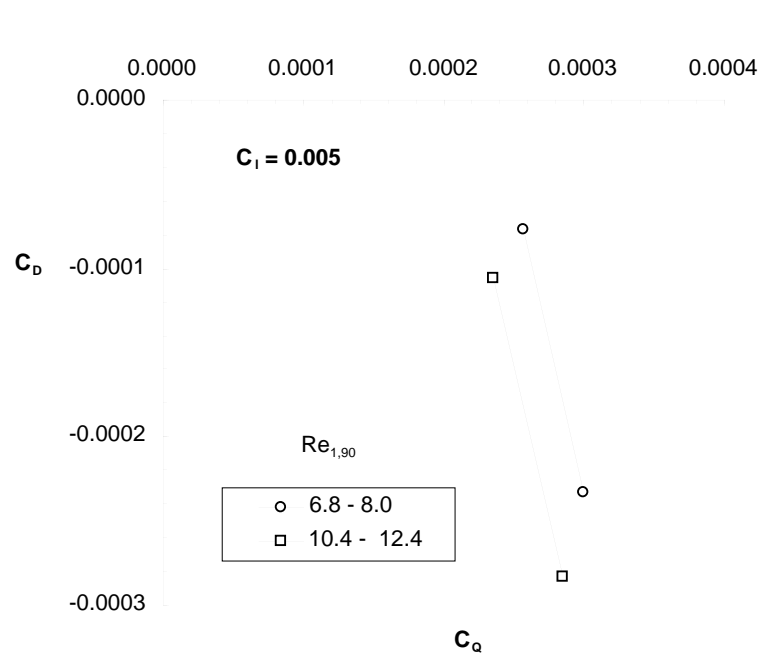

Figure 21: Effect of Reynolds number at constant Lock number for $C_{L}=0.005$.

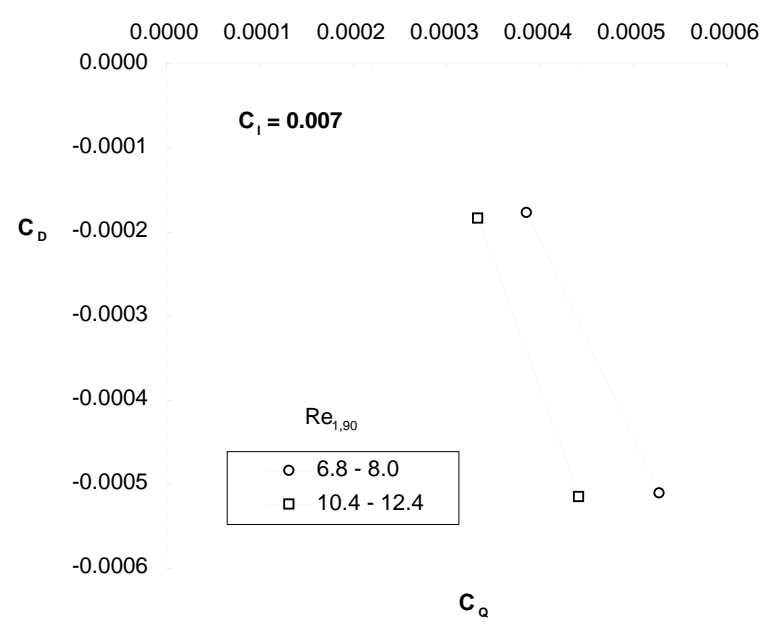

Figure 22: Effect of Reynolds number at constant Lock number for $C_{L}=0.007$.

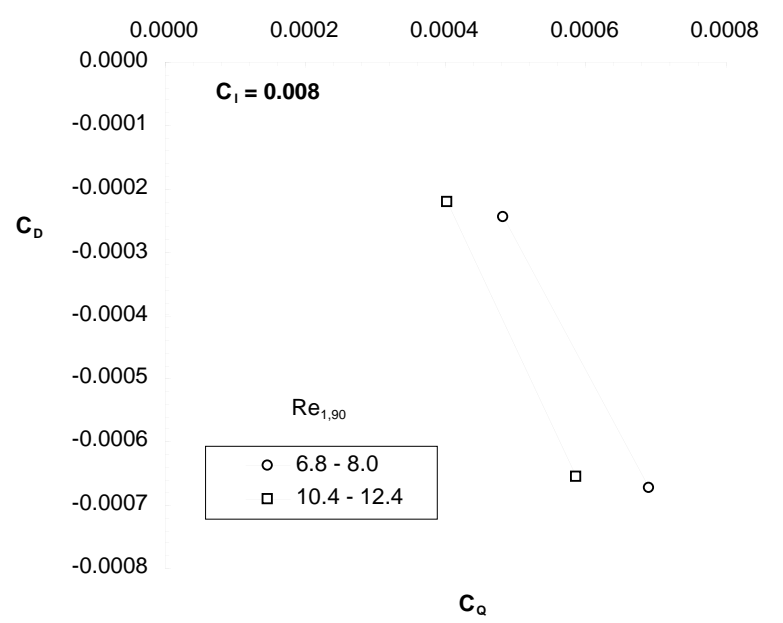

Figure 23: Effect of Reynolds number at constant Lock number for $C_{L}=0.008$. 